\title{
Chromosomal loci important for cotyledon opening under UV-B in Arabidopsis thaliana
}

\author{
Mariana Conte1, Silvia de Simone1, Susan J Simmons², Carlos L Ballaré1 and Ann E Stapleton*3
}

\begin{abstract}
Background: Understanding of the genetic architecture of plant UV-B responses allows extensive targeted testing of candidate genes or regions, along with combinations of those genes, for placement in metabolic or signal transduction pathways.

Results: Composite interval mapping and single-marker analysis methods were used to identify significant loci for cotyledon opening under UV-B in four sets of recombinant inbred lines. In addition, loci important for canalization (stability) of cotyledon opening were detected in two mapping populations. One candidate locus contained the gene HY5. Mutant analysis demonstrated that HY5 was required for UV-B-specific cotyledon opening.

Conclusions: Structured mapping populations provide key information on the degree of complexity in the genetic control of UV-B-induced cotyledon opening in Arabidopsis. The loci identified using quantitative trait analysis methods are useful for follow-up testing of candidate genes.
\end{abstract}

\section{Background}

Higher plants have complex sensory mechanisms to detect changes in the light environment [1-5]. There is evidence from physiological experiments that low levels of UV-B radiation (280-315 $\mathrm{nm}$ ) induce photomorphogenic responses in Arabidopsis thaliana [6,7] and other species $[8,9]$. Results from studies of UV-B-induced photomorphogenesis [6] and gene expression [10-12] point to the existence of a complex web of interactions involving phytochromes and cryptochromes as a part of the underlying perception, regulation and/or signaling system [5]. However, several responses induced by low-dose UV-B appear to be to some extent independent of the photoreceptors that mediate responses to visible radiation $[6,7,11,13]$. Cotyledon-opening under defined UV-B conditions is a key phenotype, as this morphological response is consistent with signalling through a photoreceptor [6]. Fluence-response curves and the independence of the cotyledon-opening response from DNA repair make this defined cotyledon-opening phenotype especially useful for genetic screens [6].

* Correspondence: stapletona@uncw.edu

${ }^{3}$ Department of Biology and Marine Biology, University of North Carolina at Wilmington, Wilmington, NC 28403 USA

Full list of author information is available at the end of the article
Screens for isolating mutants with altered sensitivity to UV-B in hypocotyls have been performed and led to the identification of uli3, a UV-B hyposensitive mutant [13], and $u v r 8$, a mutant hypersensitive to UV-B $[14,15]$. Both mutants appear to be altered in UV-B-specific signaling pathways. Photomorphogenic mutant analyses implicate the b-ZIP transcriptional factor HY5 as a component in the UV-B regulation of the expression of selected genes [11], and the COP1 gene as a regulator of HY5 and additional gene expression under UV-B [16]. Extensive analysis of UVR8 indicates that nuclear localization of the protein is important in UV-B signal transduction [17] and that UVR8 and HY5 are part of the same signal transduction pathway for gene expression responses [18].

Genome scanning is another useful approach for the isolation of loci that encode components of UV-B perception and signalling mechanisms. Genetic variability in wild ecotypes of $A$. thaliana provides an opportunity to discover genetic regions controlling phenotypic differences $[19,20]$. Genetically complex or polygenic inheritance, quantitative measurement and the availability of DNA markers allow access to quantitative trait loci (QTL) [21,22]. QTL mapping studies delimit the chromosomal regions controlling quantitative traits, and in some cases allow the identification of new alleles or causal mutations [23-25]. In the Arabidopsis model system QTL 
analysis has been performed with potentially adaptive traits like salt tolerance [26], aluminium tolerance [27], and powdery mildew disease resistance [28], as well as with developmental or physiological traits such as seed size [29], leaf architecture [30], inflorescence development [31] and growth rate [32]. It is possible to compare genetic architecture in different environments, including controlled treatment environments, although the power to detect loci will be less than the detection power for overall genetic effects [33].

Metabolic and signalling pathways can be identified from epistatic interactions [34-37], and gene interactions are widespread and important in theory and in most data sets. Epistatic interactions can explain substantial amount of variance [38], although power to detect epistasis is low in most recombinant inbred (RI) experiments with 100-200 lines. Better understanding of the genetic architecture of UV-B responses will thus be achieved by incorporation of epistatic locus interactions into the analysis.

Differences in phenotype measurements between individuals of the same RI genotype are due to random fluctuation and alleles genetically controlling stability (canalization, for developmental traits) or variance (anticanalization) $[39,40]$. Arabidopsis loci for canalization of flowering and plant growth have been found previously, by QTL mapping of RI lines [41]. Full understanding of phenotype and environmental constraints on phenotype expression require consideration of the stability of the phenotype as well as the extent of the effect of important alleles.

No QTL studies have been reported with UV-Binduced responses in Arabidopsis. In rice Sato et al. [42] have reported three QTLs controlling UV-B resistance, with a more precise mapping of the location of one of the reported QTLs [43]. Recombinant inbred lines, or in some species clones of $\mathrm{F}_{2}$ families, are the most useful for exploration of genotype and environment interactions, as the same genotype can be exposed to multiple environments [33].

In the experiments described in this paper we used four sets of RI lines to perform a QTL analysis of UV-Binduced photomorphogenesis in Arabidopsis. We measured cotyledon opening in de-etiolating seedlings as a model photomorphogenic response; under certain experimental conditions this response is induced by UV-B (but not UV-A) and the induction mechanism for cotyledon opening does not appear to involve signals derived from UV-B-induced DNA damage [6]. We used composite interval mapping to locate additive, UV-B-specific and epistatic QTL, by incorporating all the data including replicates and multiple 'environments', which in this case are UV-B treatment and control (-UV-B) growth conditions. We also analyzed these data with a straightforward single-marker method to select the most robust loci for candidate gene hypothesis testing. Loci for cotyledonopening stability were identified; these include loci at the same position as the cotyledon opening QTL and new loci that regulate stability but not extent of cotyledon opening. We combined our QTL with information from the literature to produce a list of candidate genes for further testing. Mutations in our top candidate gene, HY5, were defective in UV-B-induced cotyledon opening.

\section{Results and Discussion}

Cotyledon opening in Arabidopsis was induced specifically by low levels of UV-B, and not by control irradiations without UV-B, as illustrated in Fig. 1. Cotyledonopening angle is a UV-B-photoreceptor-induced response [6], and was thus the phenotype used for our mapping experiments. The UV-B treatment was given to young dark-grown seedlings from the RI populations as a $2.5 \mathrm{hr}$ low-fluence pulse, as previously described for this UV-B-cotyledon-opening response [6]. Statistical analysis of these $+\mathrm{UV}-\mathrm{B}$ and $-\mathrm{UV}-\mathrm{B}$ measurements allowed identification of a total of 21 loci, with 16 loci controlling cotyledon opening (14 with additive main effects and two that were epistatic-only), four loci controlling UV-B-specific cotyledon opening, and three loci controlling the stability of cotyledon-opening.

\section{Chromosomal regions affecting cotyledon opening}

A total of 16 separate loci that control cotyledon opening are significant at our stringent experiment-wise $\mathrm{P}<0.05$ threshold (Figs. 2, 3, 4, 5). Loci are identified by chromosome and map distance codes. Details of the analysis, including estimated effect sizes for contrasting alleles, are a)

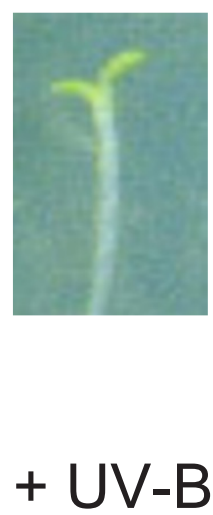

b)
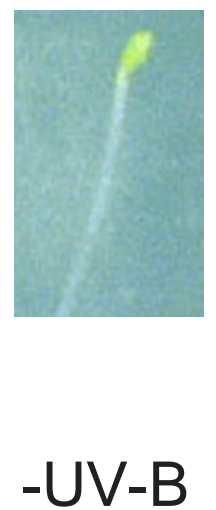

Figure 1 UV-B-induced Cotyledon Opening. Wildtype Columbia seedlings were grown and irradiated as described in Materials and Methods. a) +UV-B (cellulose di-acetate) treatment b) -UV-B (Mylar) control. 


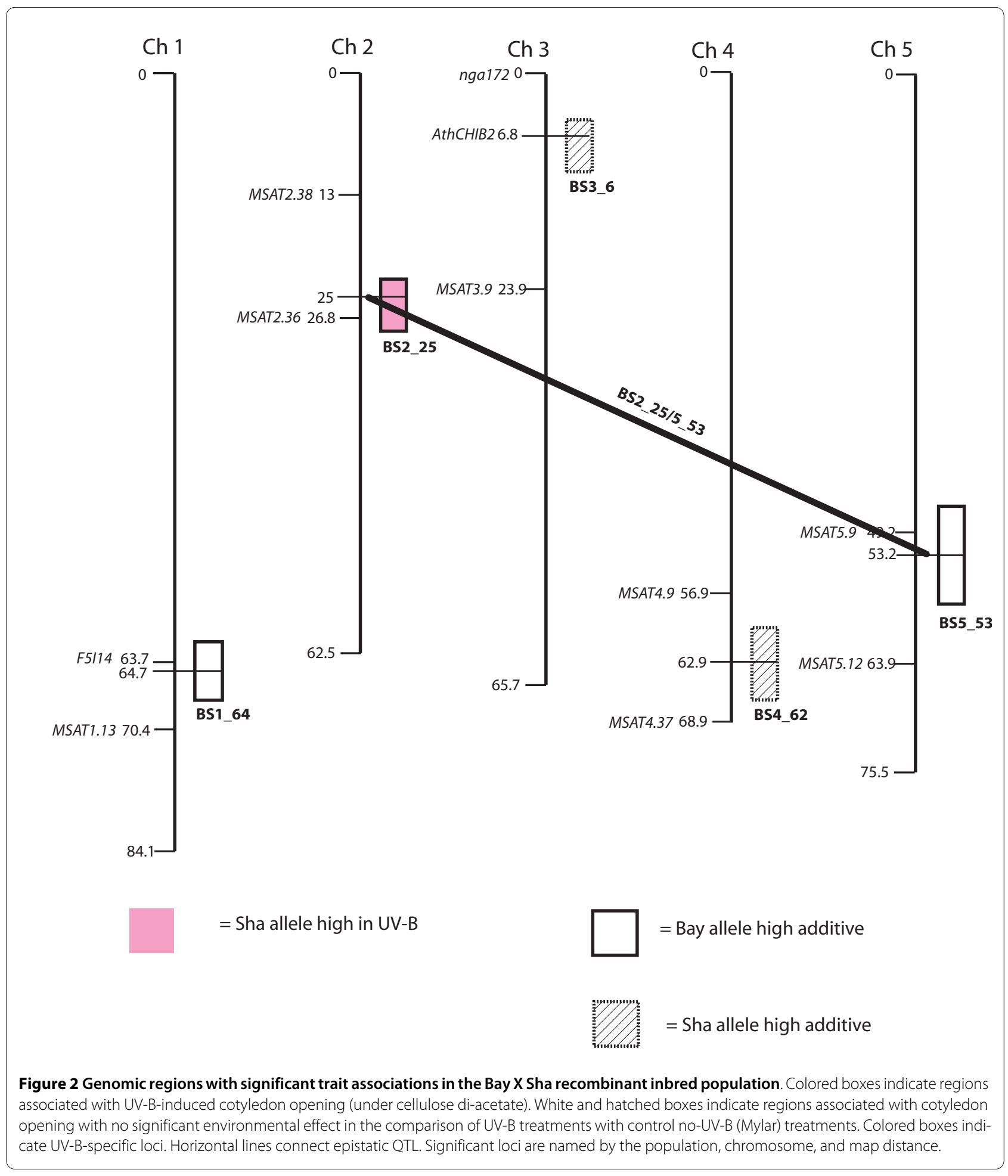

given in Additional File 1, Additional File 2, Additional File 3 and Additional File 4, arranged by locus identification code. Fifteen of these loci were also significant in the single-marker analysis. The amount of variance explained by difference in genotype differs in the four mapping populations, with LerxCol and LerxCvi having the highest heritabilities. Marker spacing is most dense in LerxCol and LerxCvi, and most sparse in BayxSha. The combination of heritability of the trait, partition of the genome into larger numbers of RI lines, and the marker parameter space determines the power to detect loci [33]; as expected, we detected the most loci in LerxCvi and Bayx- 


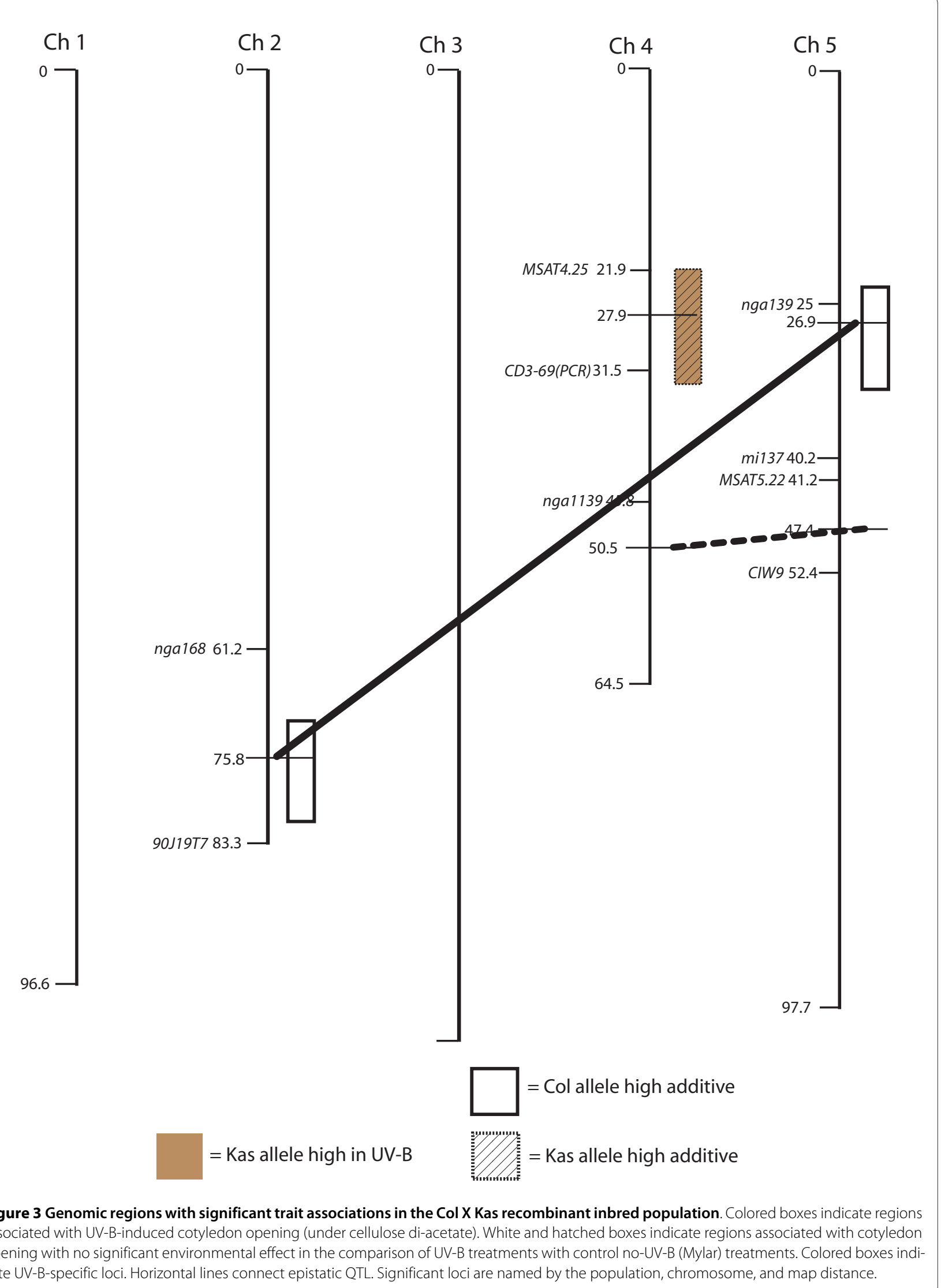




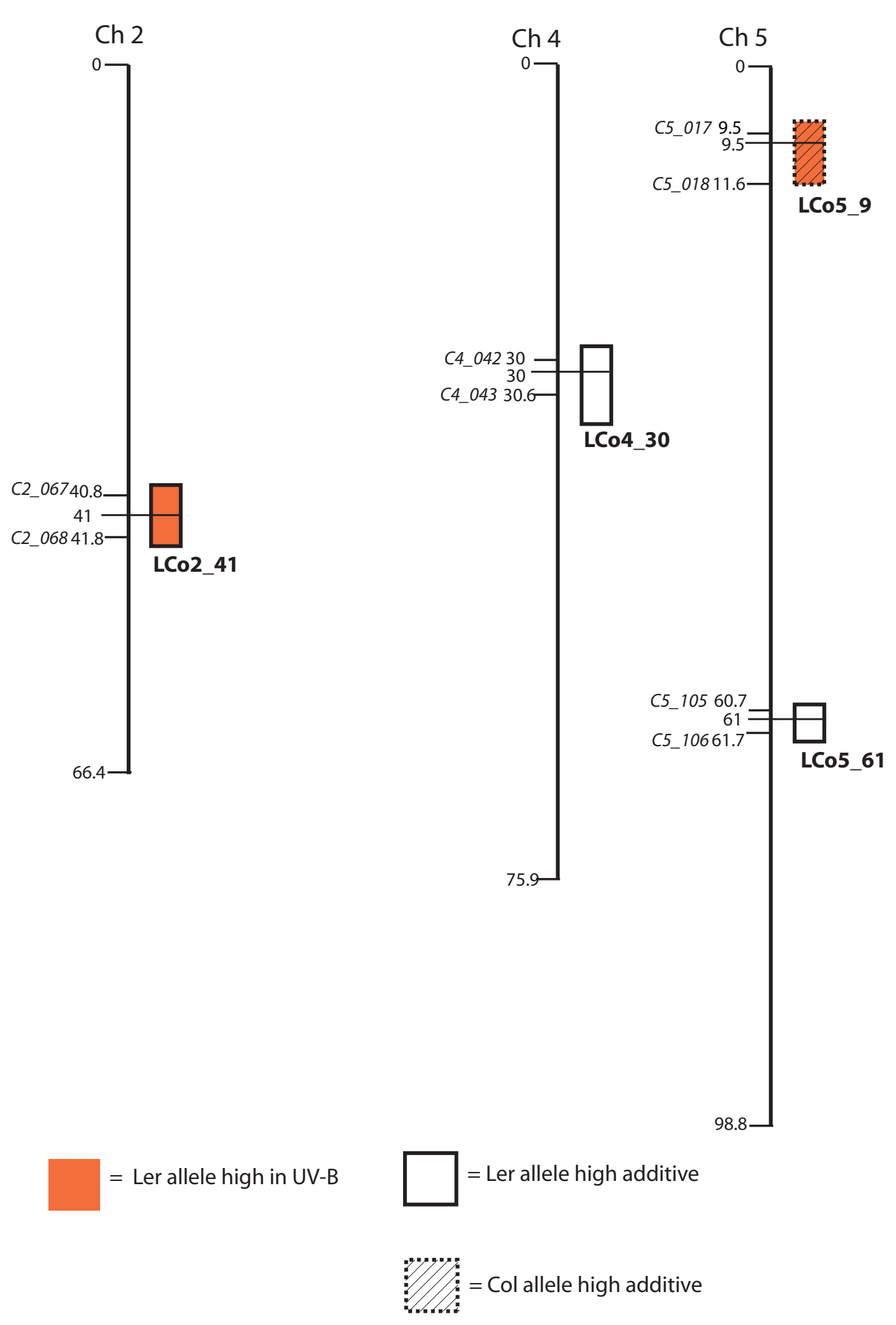

Figure 4 Genomic regions with significant trait associations in the Ler X Col recombinant inbred population. Colored boxes indicate regions associated with UV-B-induced cotyledon opening (under cellulose di-acetate). White and hatched boxes indicate regions associated with cotyledon opening with no significant environmental effect in the comparison of UV-B treatments with control no-UV-B (Mylar) treatments. Colored boxes indicate UV-B-specific loci. Horizontal lines connect epistatic QTL. Significant loci are named by the population, chromosome, and map distance. 


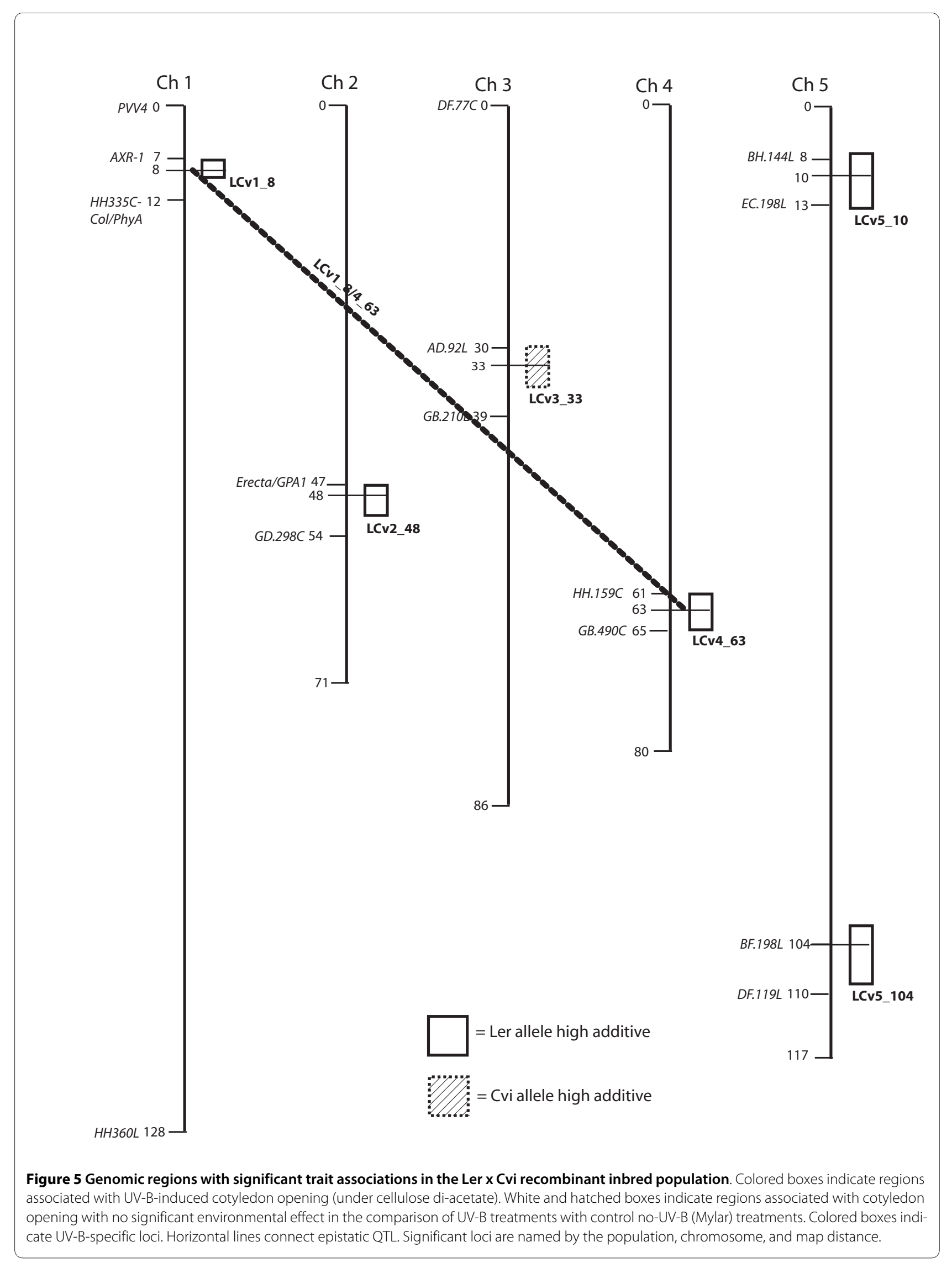


Sha populations. New genotyping methods can locate all the recombination junctions in RI populations [44], although the specific lines in our study have not yet been genotyped to this density.

\section{Comparison of epistatic interactions}

Gene interactions (epistasis) are likely to be important in explaining genetic control of phenotypic traits; however, these interactions are difficult to detect [45]. We scanned for interactions between two loci, whether or not those loci had an effect on cotyledon opening considered singly. Significant two-locus epistatic interactions were detected in the BayxSha (Fig. 2), ColxKas (Fig. 3) and LerxCvi (Fig. 5) populations. Larger mapping populations are needed for detection of multiple loci by interval mapping methods [33], and these are the larger mapping populations in our study. In BayxSha and LerxCvi the epistatic interactions had lower heritability than any individual locus, suggesting only small contributions to genetic control of cotyledon opening. In contrast, for the ColxKas population epistatic interactions are important, with heritabilities as high as those seen in individual main-effect loci (Additional File 2).

The BayxSha loci with significant epistasis also had significant main effects (Fig. 2), although the main effects were opposite in their allele contributions (Sha allele with the higher effect in BS2_25 and the Bay allele having the larger effect at BS5_53, Additional File 1). Thus, the epistatic interaction is not a simple additive effect. The estimated epistatic high allele is Bay, which suggests that the Bay allele at BS5_53 interacts equally well with either allele from the BS2_25 region, but the Sha allele at BS5_53 is more specific.

As in BayxSha, the LerxCvi epistatic loci also have single-locus main effects. In this case, both single-locus Ler alleles contribute to cotyledon opening while the epistatic alleles are from Cvi. This suggests that the Cvi alleles contribute to a pathway or complex, while the products of the Ler alleles work independently.

In the ColxKas population with the CK2_75/5_27 pair the allele effect was of the same direction as the individual locus alleles and of intermediate effect size. In this population there was also an epistatic interaction between two new loci on chromosomes 4 and 5; these new loci had no significant effect considered separately (Fig. 3). This is an example of epistasis with no main effect, which may indicate the epistatic alleles have a negative effect on their interaction partner.

Detection of interactions between loci may be useful for predicting which genes under the QTL regions are causal, as metabolic networks and gene families can cause epistasis [34-37]. The four mapping populations vary in extent of epistasis effects, indicating that particular allele interactions underlay the statistical epistasis we detected.

\section{Chromosome regions with a UV-B-specific effect}

Four loci with significant effects in the +UV-B-exposed treatment environment (cellulose di-acetate filter) were identified, BS2_25 from the BayxSha population, CK3_28 from the ColxKas population, and LC2_43 plus LC5_8 from the LerxCol population (Figs. 2, 3, 4, 5). Significant loci detected explained only about one-fourth of the genotype by environment variance. The remaining variance could be explained by presence of a number of additional small-effect loci or by epistatic interactions among more than two markers in these populations.

\section{Architecture of canalization loci}

The striking differences in the range of cotyledon opening in the RI lines (Additional File 5, Additional File 6, Additional File 7, Additional File 8, Additional File 9, Additional File 10, Additional File 11, Additional File 12) suggested that genes for the stability of the trait might be segregating in these mapping populations. Stability (canalization) is known to have a genetic component in flowering and general growth in the LerxCol and LerxCvi populations [41]. We found QTL for cotyledon-opening variance in three populations (Table 1). As expected from previous work, some QTL alleles confer both an effect on the amount of cotyledon opening and an effect on the variance. The cotyledon-opening stability QTL in LerxCol (S.LCo2_45) overlaps with the ERECTA region QTL found by Hall et al. [41]. Cotyledon-opening stability was also controlled by previously unidentified loci on Chromosome 4 in LerxCol and Chromosome 3 in BaySha (Table 1). The Chromosome 4 S.LCo4_57 locus spans 0.2 $\mathrm{Mb}$; candidate genes in this interval include a protein disulfide isomerase (At4g27080) [46] and an ascorbic acid biosynthesis gene, VTC2 [47]. The vtc2 mutant appeared to have higher variance in vegetative growth in previous studies [48]. The S.LCo2_45 erecta-region stability QTL also has an allele-specific UV-B effect; the Ler allele variance is higher in the +UV-B environment.

\section{Comparisons of chromosomal regions detected in the different populations}

We do expect to have the same alleles in geographically distinct accessions if there is strong selection. There is some overlap in the accession represented in the mapping populations, in that Ler is in two populations and $\mathrm{Col}$ in two populations. The Col common parent does not condition any common loci but in mapping populations with a Ler parent, a region in the top of Chromosome 5 is present. The additional support for these loci derived from detection in multiple populations illustrates the value of including common parents in mapping populations or using a diallel design to derive RI lines $[49,50]$. 
Table 1: Genomic Loci for Canalization

\begin{tabular}{|c|c|c|c|c|c|c|c|c|}
\hline ID & Chromosome & $\begin{array}{l}\text { Marker } \\
\text { Interval }\end{array}$ & $\begin{array}{l}\text { QTL Position } \\
\text { in } \mathrm{CM}\end{array}$ & $\begin{array}{l}\text { QTL Position } \\
\text { Range in cM }\end{array}$ & $\begin{array}{l}\text { High-Effect } \\
\text { Allele }\end{array}$ & QTL Pvalue & $h^{2}$ of QTLs & $\begin{array}{c}\text { Comparison to cotyledon } \\
\text { opening QTL }\end{array}$ \\
\hline \multicolumn{9}{|c|}{ Bay $\times$ Sha population ${ }^{1}$} \\
\hline S.BS3_10 & 3 & $\begin{array}{l}\text { ATHCHIB2- } \\
\text { MSAT3.19 }\end{array}$ & 10.8 & $4-17.8$ & Bay & $P=6.9 \times 10^{-4}$ & 0.016 & $\begin{array}{l}\text { Same locus; higher variation allele } \\
\text { has lower effect on opening. }\end{array}$ \\
\hline \multicolumn{9}{|c|}{ Ler $x$ Col population ${ }^{2}$} \\
\hline S.LCo2_45 & 2 & $\begin{array}{l}\text { C2_074- } \\
\text { C2_075 }\end{array}$ & 45.6 & $41-54$ & & & & $\begin{array}{l}\text { Locus is the same or nearby } \\
\text { (intervals overlap), has significant } \\
\text { UV-B variation effect but no additive } \\
\text { main effect. Ler allele median } \\
\text { variance is high in +UV-B round } 1 \\
(P=0.0039) \text {, and Ler allele median } \\
\text { variance is low in -UV-B round } 2 \\
(P=0.016) \text {. }\end{array}$ \\
\hline S.Lco4_57 & 4 & $\begin{array}{l}\text { C4_089- } \\
\text { C4_090 }\end{array}$ & 57.1 & $55-58$ & Col & $P=3.5 \times 10^{-5}$ & 0.043 & New locus, no UV-B-specific effect. \\
\hline
\end{tabular}




\section{Candidate genes}

We examined chromosomal regions spanned by the four UV-B-specific QTL for genes known to be important in UV-B signalling (Table 2) and for genes with UV-affected expression profiles or annotations suggesting UV function (Table 3).

We selected criteria for searching for candidate genes under QTL by considering 1) loci found in multiple populations with common alleles 2) loci with UV-B-specific effects 3) loci confirmed with both QTL Network and single-marker methods 4) size of region, and 5) the availability of additional information such as expression data on genes located near significant markers and/or annotation of genes with suggestive biochemical functions [21]. Based on these criteria, a region on the top of Chromosome 5 was selected.

\section{Candidate gene mutant hy 5 has a defect in UV-B-induced cotyledon opening}

In the LerxCol and LerxCvi populations there is a region at the top of chromosome 5 identified by QTLNetwork and single-marker analysis as controlling cotyledon opening. Examination of this region of the AGI Arabidopsis map http://www.arabidopsis.org suggested one obvious candidate gene, HY5/At5g11260, in this interval. Global gene expression experiments have implicated the HY5 gene in signal transduction from a UV-B receptor [11] and additional expression measurements have placed the UVR8 and HY5 genes in that same signal transduction pathway [18].

We examined the cotyledon-opening angles by calculating the medians split on significant parent markers for Ler $x$ Cvi and Ler $x$ Col lines. For those Ler $x$ Cvi lines that have the BH.144L Ler marker the UV-B opening angle is greater (median 101) than when Cvi allele is present (median 74); thus the Ler allele of this marker is responsive and Cvi allele is less responsive. For the Ler $\mathrm{x}$ Col population, marker C5_017 has a higher median for the Ler allele group than the $\mathrm{Col}$ allele subset, although the UV-B treatment difference is not significant in the
QTL Network analysis. This suggests that the actual locus conditioned by the Ler responsive allele is between anchor markers nga249 and nga151 on the physical map; this region includes the HY5 gene. We examined the available Ler genomic sequence [51] in this chromosomal region. There were several polymorphisms in both the coding region and in the 5' UTR region of the $H Y 5$ gene.

As Vreugdenhil et al. [52] note, the presence of multiple polymorphisms makes testing of mutants the most efficient strategy for QTL cloning in Arabidopsis. Thus, we examined UV-B-induced cotyledon opening in a HY5 mutant, hy5-215. The mutant was defective in UV-Binduced cotyledon opening (Fig. 6). This is the first identification of the role of HY5 in the low-fluence cotyledonopening responses to UV-B, which suggests that the gene expression responses controlled by UVR8 and HY5 [18] are also involved in UV-induced morphological responses in de-etiolating seedlings. In the future candidate gene insertion mutations near the other UV-specific loci that we have identified could be tested to connect cotyledon opening phenotype differences to additional specific genes.

\section{Conclusions}

Extensive characterization of morphological responses such as cotyledon-opening, when combined with genotyped structured mapping populations, allow identification of chromosomal loci and specific locus interactions important for control of the growth response. In addition to the region near $H Y 5$, we have identified several other loci associated with UV-B-induced cotyledon opening as well as loci specifying general light-induced opening.

\section{Methods}

\section{Plant material}

Four recombinant (RI) line sets of $A$. thaliana were used for QTL mapping of cotyledon opening induced by UV-B. Lines used in this work were obtained from the Arabidopsis Biological Resource Center (ABRC) in Columbus,

Table 2: Comparison of UV-B signaling gene position to QTL

\begin{tabular}{lll}
\hline UV-B signaling gene & comparison to QTLs & comments \\
\hline Uvr8 [15,18] & No overlap & \\
\hline Hy5 [11] & LCo5_9 UV-B specific QTL, also LCV5_10 & \\
\hline Hyh [18] & BS3_6 UV-B-specific QTL & Partially redundant with Hy5 \\
\hline Cop1 [16] & No overlap & Cop1 has pleiotrophic effects \\
\hline Uli3 [13] & LCV5_104 & \\
\hline
\end{tabular}


Table 3: Highest priority candidate genes under UV-B-specific QTL

\begin{tabular}{lll}
\hline QTL & candidate & comment \\
\hline LCo5_9 & HY5 & also LCv5_10 \\
\hline BS2_25 & At2g07190 hsp27-like & Large QTL region that includes CEN2 \\
\hline CK4_27 & At4g31500 RED1 & RED1 is downregulated by PHYB [67] and \\
& & has auxin phenotypes. \\
\hline LCo2_41 & At2g26710 light signaling & $\begin{array}{l}\text { Involved in multiple light signalling } \\
\text { pathways; UV-regulated expression [11] }\end{array}$ \\
\hline
\end{tabular}

Ohio http://www.arabidopsis.org. The accession numbers include CS57921 for Bay x Sha, with 165 RILs developed by [53], CS84999 for Col x Kas, with 128 RILs developed by [28], CS1899 for Ler x Col, with 99 RILs (one is redundant), developed by [54], and CS22000 for Ler x Cvi, with 162 RILs developed by [55]. The hy5-215 mutant seeds in the Columbia background were kindly provided by X.-W. Deng (Yale University).

\section{QTL experimental design}

We designed our QTL-mapping experiment following the guidelines described by Belknap [56] and Lynch and Walsh [33], with the additional priority of ensuring that populations with multiple parent ecotypes were included [57]. As our priority was detection of loci important for

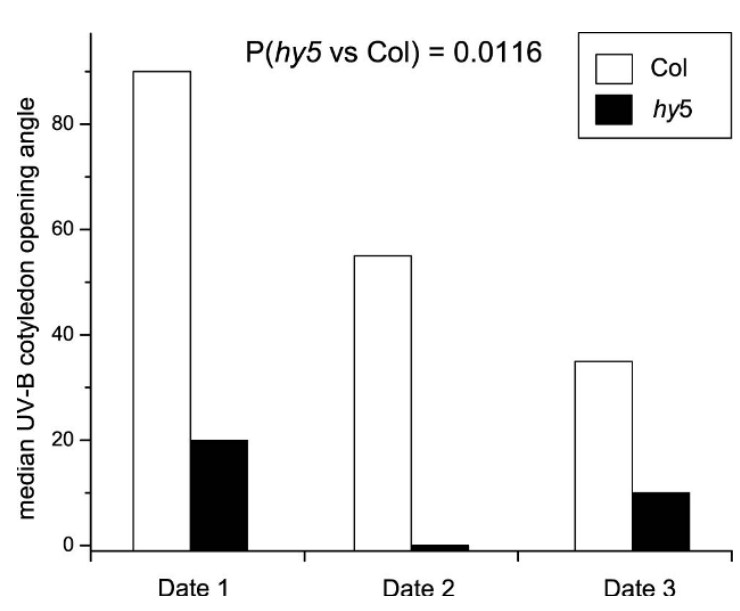

Figure 6 UV-B-induced cotyledon opening in ecotype Columbia and hy5-215. The median cotyledon opening angle for each line in each of the three separate experiments is shown. Overall $P$ value from a general linear model for the comparison of the extent of opening in the two lines is $P=0.0116$. Interactions between experiment number and line were significant. For experiment 1 , Col $n=15$, hy $5 n=15$. For experiment 2, Col $n=10$, hy $5 n=16$. For experiment $3, \operatorname{Col} n=10$ and hy $5 \mathrm{n}=10$. Pairwise permutation tests on each time point individually gave $P<0.0001$
UV-B-induced cotyledon opening, we chose to measure more replicates. This allows better estimation of effect sizes (amount of cotyledon opening conferred by the two alternative alleles at a locus), at the cost of less precise estimation of the position of the QTL on the map and less ability to detect 'small' QTL that have relatively little effect on the response [56,58]. Measurements on each RI line under each treatment (+UV-B and -UV-B) were repeated, with the two sets of measurements named round1 and round2 (Additional File 5, Additional File 6, Additional File 7, Additional File 8, Additional File 9, Additional File 10, Additional File 11, Additional File 12). Eight seeds were planted for each treatment-RI line combination for each round. The two rounds were analyzed separately, in a way similar to traditional crop QTL analyses where planting year is analyzed as a separate factor.

\section{Plant growth conditions}

Eight seeds of each RI line or 16 seeds of hy5-215 and Col wildtype $[59,60]$ were sown in $1 \mathrm{~cm}$ height plastic boxes containing $0.8 \%$ agar $(\mathrm{w} / \mathrm{v})$. Boxes were covered with UVB transparent film (Rolopac, Buenos Aires, $0.025 \mathrm{~mm}$ ) and stored for three days in darkness at $6^{\circ} \mathrm{C}$. To induce germination, seeds were exposed to an R-light pulse (30 minutes) and incubated in darkness at $25^{\circ} \mathrm{C}$ for 24 hours before being transferred to the UV-B irradiation chambers. $\mathrm{R}$ light $\left(30 \mu\right.$ moles $\left.\mathrm{m}^{-2} \mathrm{~s}^{-1}\right)$ was provided by red fluorescent tubes (40/15, Philips).

\section{UV-B treatment}

UV-B treatment and measurement of cotyledon opening were carried out as previously described [6]. Briefly, oneday-old etiolated seedlings were exposed for 3 days to a daily period of 2.5 hours of UV-B followed by a 5 minute $\mathrm{R}$ pulse. Seedlings were transferred to the dark for 24 hours and then the aperture of the cotyledons was measured on the fourth day. UV-B was provided by two UV-B 313 bulbs (Q-Panel 313, Cleveland), with a 0.1-mm-thick cellulose di-acetate film (La Casa del Celuloide, Buenos Aires) placed between the tubes and the seedlings to filter 
out the UV-C radiation emitted by the fluorescent tubes, as previously described [12,15]. UV-B level was measured with a IL1700 double-monochromator spectroradiometer (International Light, Newburyport, MA), integrating the spectral irradiance between 290 and $315 \mathrm{~nm}$. The radiometer was calibrated against a standard lamp (OL40, Optronic, Orlando, FL) in the short-wavelength range and a model 1800 calibrator (LI-COR, Lincoln, NE) for wavelengths greater than $320 \mathrm{~nm}$. In order to obtain a control treatment (-UV-B treatment), the UV-B portion of the spectrum emitted by the Q-Panel 313 bulbs was removed using a clear polyester film (Mylar-D, DuPont, Wilmington, DE; 0.1-mm thick). Neutral density filters were placed below the filters to reduce the intensity of UV to $2.25 \mu$ moles $\mathrm{m}^{-2} \mathrm{sec}^{-1}$. This photon flux was identified as a suitably low level from the action spectrum for UV-B-induced-cotyledon-opening [6]. UV spectra under each film type are provided in Additional File 13.

\section{Measurement of cotyledon opening}

Cotyledon aperture was measured with a protractor and magnifying glass. Accuracy of measurement for each assayer was checked by measuring test seedlings 6-9 times; the coefficient of variation of the measurements within and between raters was less than 5\% (data not shown). Measurements were expressed as angle between the cotyledons $\left(0\right.$ to $\left.180^{\circ}\right)$. The raw measurement data (in degrees) on all individual plants is available in Additional File 5, Additional File 6, Additional File 7, Additional File 8, Additional File 9, Additional File 10, Additional File 11 and Additional File 12, arranged by population and date of measurement.

\section{Molecular markers}

These RI lines have been characterized for molecular markers and that data was accessed from the Natural resource at http://arabidopsis.info/BrowsePage. We removed uninformative markers from our analysis by checking each adjacent marker for difference in the line distribution pattern of the markers. If the line distribution pattern in two successive markers on the chromosome was exactly the same, the second marker was removed. In order to determine if there was any artificial (non-syntenic) correlation in the particular RI line sets that we used, we performed Pearson correlations on each line set, as previously described [61], using the SAS procedure CORR. There was little artificial correlation evident in the line sets we used (data not shown).

\section{Variance phenotype}

To adjust for the large numbers of zeros in the data set, 0.5 was added to each phenotype measurement. Levene's median natural log statistic for each individual was then calculated as previously described [41,62]. Using the median form is a conservative choice as compared to use of the mean version of the Levene's statistics, as the median form is less sensitive and less susceptible to artefacts with increasing variance in larger trait measurements. The full data set was used for QTLNetwork analysis.

\section{QTL Network v. 2.0 analysis}

This composite interval mapping program [63] was accessed from http://ibi.zju.edu.cn/software/qtlnetwork/. This particular CIM mapping program was chosen as it has provision for appropriate analysis of multiple replicates of each line and for multiple environments (+UV-B and -UV-B, in our case) [64]. This program estimates effect sizes using a Bayesian fitting method [64], and chooses cofactors automatically. Program default settings (test window of $10 \mathrm{cM}$, walk speed of $1 \mathrm{cM}$ ) were used, except for deselection of the option for best genotype prediction. The experiment-wise $\mathrm{P}$ value threshold (across the whole experiment, two rounds of two treatment environments for eight replicate genotypes of each RIL in each of the four populations) was kept at $\mathrm{P}=0.05$, with 1000 permutations for $\mathrm{F}$-value threshold selection.

\section{Marker-based analysis}

A mixed model was constructed in SAS v9.1 (SAS Inc, Cary, NC) for individual marker state and UV-B treatment. Each round of the experiment was analyzed separately. A genome-wide $\mathrm{P}$ value threshold of $10^{-4}$ was chosen $[33,65]$, as the marker density in these RI line sets was not large enough to require adjustment for marker correlation [66].

\section{hy5 data analysis}

Cotyledon opening angles in mutant and Columbia wildtype were compared in individual experiments by permutation test and then in all three experiments. The modeling of all hy5 and Col measurements was performed using SAS PROC GENMOD with the angle measurements divided by ten to generate truncated values between 0 and 18 and specifying a Poisson distribution.

\section{Additional material}

Additional File 1 Details about significant quantitative trait loci from
the BayxSha mapping population.
Additional File 2 Details about significant quantitative trait loci from
the ColxKas mapping population.
Additional File 3 Details about significant quantitative trait loci from
the LerxCol mapping population.
Additional File 4 Details about significant quantitative trait loci from
the LerxCvi mapping population.
Additional File 5 All measured cotyledon-opening angles, arranged
by experimental round and RIL population in excel spreadsheet for-
mat (xIs). Each file contains angles for with both $+U V-B$ (cellulose di-ace-
tate) and -UV-B (Mylar).


Additional File 6 All measured cotyledon-opening angles, arranged by experimental round and RIL population in excel spreadsheet format (xls). Each file contains angles for with both +UV-B (cellulose di-acetate) and -UV-B (Mylar)

Additional File 7 All measured cotyledon-opening angles, arranged by experimental round and RIL population in excel spreadsheet format (xIs). Each file contains angles for with both +UV-B (cellulose di-acetate) and -UV-B (Mylar).

Additional File 8 All measured cotyledon-opening angles, arranged by experimental round and RIL population in excel spreadsheet format (xIs). Each file contains angles for with both +UV-B (cellulose di-acetate) and -UV-B (Mylar).

Additional File 9 All measured cotyledon-opening angles, arranged by experimental round and RIL population in excel spreadsheet format (xls). Each file contains angles for with both +UV-B (cellulose di-acetate) and -UV-B (Mylar).

Additional File 10 All measured cotyledon-opening angles, arranged by experimental round and RIL population in excel spreadsheet format (xIs). Each file contains angles for with both +UV-B (cellulose di-acetate) and -UV-B (Mylar).

Additional File 11 All measured cotyledon-opening angles, arranged by experimental round and RIL population in excel spreadsheet format (xls). Each file contains angles for with both +UV-B (cellulose di-acetate) and -UV-B (Mylar).

Additional File 12 All measured cotyledon-opening angles, arranged by experimental round and RIL population in excel spreadsheet format (xls). Each file contains angles for with both +UV-B (cellulose di-acetate) and -UV-B (Mylar).

Additional File 13 UV Spectra. Additional description and a figure showing the spectral output of the UV313 bulbs and the irradiance under the Mylar-D and cellulose di-acetate filters used for -UV-B and +UV-B treatment of cotyledons.

\section{Authors' contributions}

This project was conceived by AS, who provided grant support for seed purchase and travel. MC and SdS grew the plants and measured cotyledon opening. SS and AS carried out the data analysis. MC, AS and SS wrote the manuscript; CB edited the manuscript, provided support for MC and SdS, and hosted AS in his lab. All authors read and approved the final manuscript.

\section{Acknowledgements}

We gratefully acknowledge support from the Women's International Science Collaboration (WISC--a program of the American Association for the Advancement of Science) to A. S. in 2003-2004, and from Agencia Nacional de Promoción Cientifica y Tecnológica and Universidad de Buenos Aires to C. B. The funders had no role in study design, data collection and analysis, decision to publish, or preparation of the manuscript. We are grateful to James E. Blum, Department of Mathematics and Applied Statistics at UNCW, who wrote the SAS code for correlation analysis and html display. We also thank Carlos Mazza, IFEVA, for providing the UV bulb spectra.

\section{Author Details}

IInstituto de Investigaciones Fisiológicas y Ecológicas Vinculadas a la Agricultura, Consejo Nacional de Investigaciones Científicas y Técnicas and Universidad de Buenos Aires, C1417 DSE Buenos Aires, Argentina, 2Department of Mathematics and Statistics, University of North Carolina at Wilmington, Wilmington, NC 28403 USA and 3Department of Biology and Marine Biology, University of North Carolina at Wilmington, Wilmington, NC 28403 USA

Received: 3 March 2010 Accepted: 16 June 2010

Published: 16 June 2010

\section{References}

1. Ballaré CL: Illuminated behaviour. Phytochrome as a key regulator of light foraging and plant anti-herbivore defence. Plant Cell Environ 2009, 32:713-725.

2. Frohnmeyer $\mathrm{H}$, Staiger D: Ultraviolet-B radiation-mediated responses in plants. Balancing damage and protection. Plant Physio/ 2003, 133(4):1420-1428
3. Ulm R, Nagy F: Signalling and gene regulation in response to ultraviolet light. Curr Opin Plant Biol 2005, 8:477-482.

4. Franklin KA, Larner VS, Whitelam GC: The signal transducing photoreceptors of plants. Int J Devel Biol 2005, 49:653-664.

5. Jenkins Gl: Signal transduction in responses to UV-B radiation. Ann ReV Plant Bio 2009, 60:407-431.

6. Boccalandro HE, Mazza CA, Mazzella MA, Casal JJ, Ballaré CL: Ultraviolet B radiation enhances a phytochrome-B-mediated photomorphogenic response in Arabidopsis. Plant Physiol 2001, 126:780-788.

7. Kim BC, Tennessen DJ, Last RL: UV-B-induced photomorphogenesis in Arabidopsis thaliana. Plant J 1998, 16:667-674.

8. Ballare CL, Barnes PW, Flint SD: Inhibition of hypocotyl elongation by ultraviolet-B radiation in de-etiolating tomato seedlings. I. The photoreceptor. Physiol Plant 1995, 93:584-592.

9. Ballaré $C L$, Barnes PW, Kendrick RE: Photomorphogenic effects of UV-B radiation on hypocotyl elongation in wild type and stablephytochrome-deficient mutant seedlings of cucumber. Physiol Plant 1991, 83:652-658.

10. Jenkins Gl, Fuglevand G, Christie JM: UV-B perception and signal transduction. In Plants and UV-B Responses to Environmental Change Volume 64. Edited by: Lumsden P. Cambridge: Cambridge University Press; 1997:135-156.

11. Ulm R, Baumann A, Oravecz A, Mate Z, Adam E, Oakeley EJ, Schafer E, Nagy F: Genome-wide analysis of gene expression reveals function of the bZIP transcription factor HY5 in the UV-B response of Arabidopsis. Proc Natl Acad Sci USA 2004, 101:1397-1402.

12. Wade HK, Bibikova TN, CValentine WJ, Jenkins Gl: Interactions within a network of phytochrome, cryptochrome and UV-B phototransduction pathways regulate chalcone synthase gene expression in Arabidopsis leaf tissue. Plant J 2001, 25:675-685.

13. Suesslin C, Frohnmeyer $\mathrm{H}$ : An Arabidopsis mutant defective in UV-B light-mediated responses. Plant J 2003, 33:591-601.

14. Brown BA, Cloix C, Jiang GH, Kaiserli E, Herzyk P, Kliebenstein DJ, Jenkins GI: A UV-B-specific signaling component orchestrates plant UV protection. Proc Natl Acad Sci USA 2005, 102:18225-18230.

15. Kliebenstein DJ, Lim JE, Landry LG, Last RL: Arabidopsis UVR8 regulates ultraviolet-B signal transduction and tolerance and contains sequence similarity to human regulator of chromatin condensation 1. Plant Physiol 2002, 130:234-243.

16. Oravecz A, Baumann A, Mate Z, Brzezinska A, Molinier J, Oakeley EJ, Adam E, Schafer E, Nagy F, UIm R: CONSTITUTIVELY PHOTOMORPHOGENIC1 is required for the UV-B response in Arabidopsis. Plant Cell 2006, 18:1975-1990.

17. Kaiserli E, Jenkins Gl: UV-B promotes rapid nuclear translocation of the Arabidopsis UV-B specific signaling component UVR8 and activates its function in the nucleus. Plant Cell 2007, 19:2662-2673.

18. Brown BA, Jenkins GI: UV-B signaling pathways with different fluencerate response profiles are distinguished in mature Arabidopsis leaf tissue by requirement for UVR8, HY5, and HYH. Plant Physiol 2008, 146:576-588.

19. Alonso-Blanco C, Koornneef M: Naturally occurring variation in Arabidopsis: an underexploited resource for plant genetics. Trends Plant Sci 2000, 5:22-29.

20. Tonso SJ, Alonso-Blanco C, Koornneef M: Gene function beyond the single trait: natural variation, gene effects, and evolutionary ecology in Arabidopsis thaliana. Plant Cell Environ 2005, 28:2-20.

21. Borevitz JO: Genomics tools for QTL analysis and gene discovery. Curr Opin Plant Biol 2004, 7:132-136.

22. Shindo C, Bernasconi G, Hardtke CS: Natural genetic variation in Arabidopsis: tools, traits and prospects for evolutionary ecology. Ann Bot 2007, 99:1043-1054.

23. Botto JF, Alonso-Blanco C, Garzaron I, Sanchez RA, Casal JJ: The Cape Verde Islands allele of cryptochrome 2 enhances cotyledon unfolding in the absence of blue light in Arabidopsis. Plant Physiol 2003 133:1547-1556.

24. El-Din S El-Assal, Alonso-Blanco C, Peeters AJ, Raz V, Koornneef M: A QTL for flowering time in Arabidopsis reveals a novel allele of CRY2. Nat Genet 2001, 29:435-440.

25. Alonso-Blanco C, Aarts MGM, Bentsink L, Keurentjes JJB, Reymond M, Vreugdenhil D, Koornneef $M$ : What has natural variation taught us about plant development, physiology, and adaptation? Plant Cell 2009, 21:1877-1896. 
26. Quesada V, Garcia-Martinez S, Piqueras P, Ponce MR, Micol JL: Genetic architecture of $\mathrm{NaCl}$ tolerance in Arabidopsis. Plant Physiol 2002, 130:951-963.

27. Kobayashi Y, Koyama $\mathrm{H}$ : QTL analysis of $\mathrm{Al}$ tolerance in recombinant inbred lines of Arabidopsis thaliana. Plant Cell Physiol 2002, 43:1526-1533.

28. Wilson IW, Schiff CL, Hughes DE, Somerville SC: Quantitative trait loci analysis of powdery mildew disease resistance in the Arabidopsis thaliana accession Kashmir-1. Genetics 2001, 158:1301-1309.

29. Alonso-Blanco C, Blankestijn-de H Vries, Hanhart CJ, Koornneef M: Natural allelic variation at seed size loci in relation to other life history traits of Arabidopsis thaliana. Proc Natl Acad Sci USA 1999, 96:4710-4717.

30. Perez-Perez JM, Serrano-Cartagena J, Micol JL: Genetic analysis of natural variations in the architecture of Arabidopsis thaliana vegetative leaves. Genetics 2002, 162:893-915.

31. Ungerer MC, Halldorsdottir SS, Modliszewski JL, Mackay TFC, Purugganan M: Quantitative trait loci for inflorescence development in Arabidopsis thaliana. Genetics 2002, 160:1133-1151.

32. El-Lithy ME, Clerkx EJ, Ruys GJ, Koornneef M, Vreugdenhil D: Quantitative trait locus analysis of growth-related traits in a new Arabidopsis recombinant inbred population. Plant Physiol 2004, 135:444-458.

33. Lynch M, Walsh B: Genetics and Analysis of Quantitative Traits. Sunderland, MA: Sinauer Associates, Inc.; 1998.

34. Gjuvsland AB, Hayes BJ, Omholt SW, Carlborg O: Statistical epistasis is a generic feature of gene gegulatory networks. Genetics 2007, 175:411-420.

35. Rowe HC, Hansen BG, Halkier BA, Kliebenstein DJ: Biochemical networks and epistasis shape the Arabidopsis thaliana metabolome. Plant Cell 2008, 20:1199-1216.

36. Stich B, Yu J, Melchinger AE, Piepho HP, Utz HF, Maurer HP, Buckler ES: Power to detect higher-order epistatic interactions in a metabolic pathway using a new mapping strategy. Genetics 2007, 176:563-570.

37. Kliebenstein D: Advancing genetic theory and application by metabolic quantitative trait analysis. Plant Cell 2009, 21:1637-1646.

38. Malmberg RL, Held S, Waits A, Mauricio R: Epistasis for fitness-related quantitative traits in Arabidopsis thaliana grown in the field and in the greenhouse. Genetics 2005, 171:2013-2027.

39. de Visser JAGM, Hermisson J, Wagner GP, Meyers LA, Bagheri-Chaichian H, Blanchard JL, Chao L, Cheverud JMSF, Elena SFW, Fontana W, et al: PERSPECTIVE: Evolution and detection of genetic robustness. Evolution 2003, 57:1959-1972.

40. Gibson G, Dworkin I: Uncovering cryptic genetic variation. Nat Rev Genet 2004, 5:681-690.

41. Hall MC, Dworkin I, Ungerer MC, Purugganan M: Genetics of microenvironmental canalization in Arabidopsis thaliana. Proc Natl Acad Sci 2007, 104:13717-13722.

42. Sato T, Ueda T, Fukuta Y, Kumagai T, Yano M: Mapping of quantitative trait loci associated with ultraviolet-B resistance in rice (Oryza sativa L.). Theor App/ Genet 2003, 107:1003-1008.

43. Ueda T, Sato T, Numa H, Yano M: Delimitation of the chromosomal region for a quantitative trait locus, qUVR-10, conferring resistance to ultraviolet-B radiation in rice (Oryza sativa L.). Theor Appl Genet 2004, 108:385-391.

44. Gupta PK, Rustgi S, Mir RR: Array-based high-throughput DNA markers for crop improvement. Heredity 2008, 101:5-18.

45. Le Rouzic A, Carlborg Ö: Evolutionary potential of hidden genetic variation. Trends Ecol Evol 2008, 23:33-37.

46. Park SK, Jung YJ, Lee JR, Lee YM, Jang HH, Lee SS, Park JH, Kim SY, Moon JC, Lee SY, et al:: Heat-shock and redox-dependent functional switching of an h-Type Arabidopsis thioredoxin from a disulfide reductase to a molecular chaperone. Plant Physiol 2009, 150:552-561.

47. Conklin PL, Saracco SA, Norris SR, Last RL: Identification of ascorbic aciddeficient Arabidopsis thaliana mutants. Genetics 2000, 154:847-856.

48. Muller-Moule P, Golan T, Niyogi KK: Ascorbate-deficient mutants of Arabidopsis grow in high light despite chronic photooxidative stress. Plant Physiol 2004, 134:1163-1172.

49. Jannink JL, Jansen RC: Mapping epistatic quantitative trait loci with one-dimensional genome searches. Genetics 2001, 157:445-454

50. Simon M, Loudet O, Durand S, Berard A, Brunel D, Sennesal FX, DurandTardif M, Pelletier G, Camilleri C: Quantitative trait loci mapping in five new large recombinant inbred line populations of Arabidopsis thaliana genotyped with consensus single-nucleotide polymorphism markers. Genetics 2008, 178:2253-2264.

51. Jander G, Norris SR, Rounsley SD, Bush DF, Levin IM, Last RL: Arabidopsis map-based cloning in the post-genome era. Plant Physiol 2002, 129(2):440-450

52. Vreugdenhil D, Koornneel M, Sergeeva L: Use of QTL analysis in physiological research. Russ J Plant Physiol 2007, 54:10-15.

53. Loudet O, Chaillou S, Camilleri C, Bouchez D, Daniel-Vedele F: Bay-0 x Shadara recombinant inbred line population: a powerful tool for the genetic dissection of complex traits in Arabidopsis. Theor App/ Genet 2002, 104:1173-1184.

54. Lister C, Dean C: Recombinant inbred lines for mapping RFLP and phenotypic markers in Arabidopsis thaliana. Plant J 1993, 4:745-750.

55. Alonso-Blanco C, Peeters AJ, Koornneef M, Lister C, Dean C, van den N Bosch, Pot J, Kuiper MT: Development of an AFLP based linkage map of Ler, Col and Cvi Arabidopsis thaliana ecotypes and construction of a Ler/Cvi recombinant inbred line population. Plant J 1998, 14:259-271.

56. Belknap JK: Effect of within-strain sample size on QTL detection and mapping using recombinant inbred mouse strains. Behav Genet 1998 28:29-38.

57. Cavanagh C, Morell M, Mackay I, Powell W: From mutations to MAGIC: resources for gene discovery, validation and delivery in crop plants. Curr Opin Plant Biol 2008, 11:215-221.

58. Vales M, Schön C, Capettini F, Chen X, Corey A, Mather D, Mundt C, Richardson K, Sandoval-Islas J, Utz H, et al:: Effect of population size on the estimation of QTL: a test using resistance to barley stripe rust. Theor App/ Genet 2005, 111:1260-1270.

59. Ahmad M, Cashmore AR: HY4 gene of A. thaliana encodes a protein with characteristics of blue-light photoreceptor. Nature 1993, 366:162-166.

60. Briggs WR, Christie JM: Phototropins 1 and 2: versatile plant blue-light receptors. Trends Plant Sci 2002, 7:204-210.

61. Williams RW, Gu J, Qi S, Lu L: The genetic structure of recombinant inbred mice: high-resolution consensus maps for complex trait analysis. Genome Biol 2001, 2(11):RESEARCH0046.

62. Schultz B: Levene's test for relative variation. Systemat Zoo/ 1985 34(4):449-456

63. Yang J, Hu C, Hu H, Yu R, Xia Z, Ye X, Zhu J: QTLNetwork: mapping and visualizing genetic architecture of complex traits in experimental populations. Bioinformatics 2008, 24:721-723.

64. Yang J, Zhu J, Williams RW: Mapping the genetic architecture of complex traits in experimental populations. Bioinformatics 2007, 23:1527-1536.

65. Lander ES, Botstein D: Mapping mendelian factors underlying traits using RFLP linkage maps. Genetics 1989, 121:185-199.

66. Cheverud JM: A simple correction for multiple comparisons in interval mapping genome scans. Heredity 2001, 87:52-58.

67. Wagner D, Hoecker U, Quail PH: RED1 is necessary for phytochrome Bmediated red light-specific signal transduction in Arabidopsis. Plant Cell 1997, 9:731-743.

doi: 10.1186/1471-2229-10-112

Cite this article as: Conte et al., Chromosomal loci important for cotyledon opening under UV-B in Arabidopsis thaliana BMC Plant Biology 2010, 10:112

\section{Submit your next manuscript to BioMed Central and take full advantage of:}

- Convenient online submission

- Thorough peer review

- No space constraints or color figure charges

- Immediate publication on acceptance

- Inclusion in PubMed, CAS, Scopus and Google Scholar

- Research which is freely available for redistribution 